ARTIGO ORIGINAL

\title{
Índice de Massa Corpórea, Competência Social e Problemas de Comportamento de Crianças e Cuidadores ${ }^{1}$
}

\author{
Doralice Oliveira Pires Dias ${ }^{2}$ \\ Sônia Maria Mello Neves \\ Daniela Sacramento Zanini \\ Pontificia Universidade Católica de Goiás
}

\begin{abstract}
RESUMO - O objetivo deste estudo foi avaliar a relação entre competências sociais, problemas de comportamento e Índice de Massa Corpórea (IMC) das crianças participantes de um programa de intervenção multidisciplinar para excesso de peso da família. Participaram do estudo 22 cuidadores e 26 crianças. O CBCL e o ASR foram aplicados antes da intervenção. Resultados mostraram relações significativas entre problemas de comportamento internalizantes das crianças e seu IMC, e relações entre problemas de comportamento de cuidadores e de crianças. Sugere-se que o problema de comportamento das crianças seja uma variável mediacional entre o problema de comportamento dos cuidadores e o IMC das crianças, sendo assim necessário o desenvolvimento de intervenções para o excesso de peso que intervenham nos problemas de comportamento de ambos.
\end{abstract}

Palavras-chave: obesidade, controle do peso, distúrbios do comportamento, competência, habilidades sociais

\section{Body Mass Index, Social Competence and Behavioral Problems of Children and Caregivers}

\begin{abstract}
This study aimed to investigate the relationship between social competences, behavioral problems, competences, and Body Mass Index (BMI) of children who participated in a multidisciplinary intervention program developed to deal with overweight of families. 22 caregivers and 25 children participated. Before the start of the intervention program the CBCL and ASR were administrated. The results show a significant relationship between the child's internalization of behavioral problems and the BMI as well as a relationship between the child's and the caregivers behavioral problems. The results suggest that the child's behavioral problem is a mediating variable between the caregiver's behavioral problem and the child's BMI. Therefore, the development of intervention programs of the overweight problem dealing with the behavioral problems of both is necessary.
\end{abstract}

Keywords: obesity, weight-control, behavior disorders, competence, social skills

A família é o contexto primário propiciador do desenvolvimento do repertório comportamental das crianças. A aprendizagem, nesse contexto, dá-se através do modelo de comportamento dos pais, assim como das consequências fornecidas aos seus comportamentos. Desse modo, a obesidade dos pais pode ser uma importante variável para o desenvolvimento da obesidade infantil, pois, nesse caso, fatores genéticos e ambientais estão em ação (Epstein, Valoski, Wing, \& McCurley, 1990).

De acordo com Tiffin, Arnott, Moore e Summerbell (2011), há muitas razões plausíveis pelas quais o excesso de peso pode estar associado ao ajuste psicológico pobre, entre essas o impacto da obesidade sobre a autoestima e a confiança social, o efeito direto dos hormônios e alterações metabólicas na função do cérebro, mudanças no comportamento alimentar e nos níveis de atividade física (que podem ser consequência de humor deprimido) e ganho de peso secundário ao uso de medicamentos psiquiátricos, quando for o caso.

Vários estudos realizados com o intuito de verificar relações entre as dificuldades psicológicas e a obesidade, utilizando os inventários do System of Empirically

1 Este artigo é parte da dissertação de mestrado da primeira autora

2 Programa de Pós Graduação Stricto Senso em Psicologia, Campus I, PUC Goiás, Área IV, Bloco A, $1^{\circ}$ andar, Avenida Universitária, ${ }^{\circ} 1440$, Setor Universitário, Goiânia, Goiás, Brasil. CEP 74.810-210 E-mail: doralicepires@hotmail.com
Based Assessment (ASEBA), identificam problemas de comportamento em adultos e crianças. Entre os inventários ASEBA, são conhecidos o Child Behavior Check List (CBCL), que avalia problemas de comportamento de crianças (Achenbach \& Rescorla, 2001), e o Adult Self Report (ASR), que avalia problemas de comportamento de adultos (Achenbach \& Rescorla, 2003).

Estudos com o objetivo de correlacionar problemas de comportamento e obesidade em adultos utilizando o ASR não foram encontrados na base de dados PUBMED, em pesquisa realizada em 2013, por meio das palavras-chaves Adult Self Report, ASR e Obesity. Há registros da utilização desse instrumento em estudos sobre Transtorno de Déficit de Atenção (Rösler, Retz, Thome, Schneider, Stieglitz, \& Falkai, 2008), na avaliação de problemas de comportamento em adultos que foram submetidos a transplante renal quando crianças (Haavisto, Jalanko, Sintonen, Holmberg, \& Qvist, 2006) e na associação entre problemas de comportamento de pais e problemas de comportamento das crianças (Sanger, MacLean, \& Slyke 1992). Entre os estudos que fizeram uso do CBCL para verificar possíveis correlações entre obesidade e dificuldades internalizantes e externalizantes em crianças, são conhecidos os de Braet, Mervielde e Vandereycken (1997), Israel e Shapiro (1985), Luiz, Gorayeb e Libertatore (2010) e Sousa e Moraes (2011).

O estudo de Braet et al. (1997), realizado na Bélgica, explorou a relação entre obesidade e ajustamento psicossocial 
em 139 crianças obesas e 150 não obesas, com idades entre 9 e 12 anos. As crianças preencheram uma Escala de Competências e seus pais completaram o CBCL. Todas as crianças obesas relataram mais autopercepções corporais negativas e maiores índices de baixa autoestima, assim como, de acordo com seus pais, demonstravam mais problemas de comportamento do que seus pares não obesos. Por conseguinte, a conclusão dos autores é que as crianças obesas parecem demonstrar maior perfil psicossocial de risco.

Outro estudo que constatou a relação entre obesidade e problemas de comportamento foi realizado no Brasil, por Luiz et al. (2010). Através do CBCL e do Inventário de Depressão Infantil (CDI), compararam depressão, distúrbios de comportamento e competência social em crianças obesas e não obesas, de idades entre 7 e 13 anos. Verificou-se que, no grupo de crianças obesas, havia mais distúrbios externalizantes e internalizantes do que no grupo de não obesas. Essa diferença foi especialmente significativa para os problemas internalizantes, mais especificamente para a síndrome de depressão. Em relação à competência social, constatou-se associação entre déficits de competência social e obesidade.

Sousa e Moraes (2011), também em estudo realizado no Brasil, compararam sintomas de internalização e externalização em crianças e adolescentes com e sem excesso de peso. Os resultados da avaliação, por meio do CBCL, de 88 indivíduos -53 deles com excesso de peso e 35 eutróficos (peso adequado), de idades entre 6 e 18 anos - mostraram sintomas de internalização em 14 indivíduos com excesso de peso e em 4 eutróficos, bem como sintomas de externalização em 9 indivíduos com excesso de peso e em 2 eutróficos. Os autores concluíram, nesse estudo, que os sintomas psiquiátricos prevaleceram nos indivíduos com excesso de peso. Dessa forma, quanto maior o Índice de Massa Corpórea (IMC), maior o risco do desenvolvimento de sintomas emocionais. No entanto, não há consenso na literatura sobre associações entre problemas psicológicos, tais como baixa autoestima e problemas comportamentais (internalizantes ou externalizantes), e obesidade (Anderson, He, Schoppe-Sullivan, \& Must 2010; Baile \& González, 2011; Friedman \& Brownell, 1995; Stunkard, Myles, Faith, \& Allison, 2003; Wadden, Foster, Brownell, \& Finley 1984).

Friedman e Brownell (1995) realizaram uma busca na literatura e verificaram divergências entre os estudos. Alguns estudos mostraram que a obesidade aumenta o risco para o desenvolvimento de sintomas depressivos, ao passo que outros indicaram que a obesidade não tem influência no risco para depressão. O estudo de Wadden et al. (1984), por exemplo, mostrou que os escores de autoestima de crianças obesas estavam dentro da normalidade quando comparados com os da população em geral. E, no estudo desenvolvido por Anderson et al. (2010), em que mães responderam ao CBCL, correlações entre mais altos IMC e problemas externalizantes prevaleceram na amostra de 1.364 crianças de 2 a 12 anos, com e sem excesso de peso,

Problemas comportamentais parecem estar relacionados, também, com dificuldades na adesão e resultados no tratamento da obesidade. Estudo de Epstein Wisniewski e Weng (1994), que avaliou crianças de 8 a 11 anos submetidas a um tratamento comportamental do excesso de peso, mostrou, através do CBCL, que crianças com problemas de comportamento apresentaram piores resultados.

Napolitano e Hayes (2011) acompanharam 247 mulheres com idade média de 47 anos em um programa que visava à mudança de peso através da atividade física. Para tanto, foram utilizadas a Escala de Percepção de Estresse Perceived Stress Scale (PSS) -, que avalia o grau em que a pessoa identifica diferentes situações como estressantes, e uma escala de depressão denominada The Center for Epidemiologic Studies Depression Scale (CES-D), que avalia, mediante autorrelato, os sintomas depressivos. Os resultados apontaram que, quanto menos as pessoas percebiam as situações como estressantes e quanto maior o engajamento nas atividades físicas, melhores eram os resultados na perda de peso.

Em Portugal, o estudo de Vieira et al. (2011) avaliou a associação entre resultados do tratamento da obesidade e bem-estar psicológico (autoestima, autoconceito, autoeficácia), depressão e ansiedade. Participaram do estudo 239 mulheres com média de idade de 37.6 anos. Menores escores de ansiedade e depressão assim como altos escores de autoestima, autoconceito e autoeficácia estiveram relacionados com melhores resultados no tratamento.

Intervenções para o tratamento da obesidade que focam a construção de habilidades demonstram ser eficazes. O estudo realizado, nos Estados Unidos, por Myers, Hollie, Raynor e Epstein (1998) avaliou problemas de comportamento de 116 crianças obesas de 8 a 12 anos de idade, como também de suas mães e mostrou resultados significativos após a implementação de um programa direcionado às crianças e suas mães que, além de explorar várias formas de aumentar a atividade física e diminuir a ingestão de alimentos calóricos, interviu na construção de habilidades de solução de problemas. As melhoras no estado psicológico das mães estiveram relacionadas às melhoras no ajustamento psicossocial dos filhos em todas as escalas de problemas. Os resultados de perda de peso nas crianças, por sua vez, mantiveram-se por até um ano (último encontro de follow up).

Buscando entender melhor a relação entre competências sociais e problemas de comportamento de cuidadores e crianças com excesso de peso, o presente trabalho teve como objetivo avaliar a relação entre problemas de comportamento, competências e o IMC das crianças participantes de um programa de intervenção multidisciplinar que abordava o problema de excesso de peso da família. Objetivou-se, também, verificar se os problemas de comportamento e competências de cuidadores e crianças estavam relacionados com o IMC e com a adesão da criança.

\section{Método}

\section{Participantes}

Participaram deste estudo (a) 22 cuidadores -18 do sexo feminino e 4 do sexo masculino -, com idades entre 30 e 60 anos $(M=43,12, D P=7,15)$ e IMC entre 26,00 e 45,53 $\mathrm{kg} / \mathrm{cm}^{2}(M=31,29, D P=4,86)$, todos indicando sobrepeso e obesidade; e (b) 26 crianças - 17 do sexo masculino e 9 sexo 
feminino - de idades entre 7 e 12 anos $(M=9,12, D P=1,63)$ e IMC entre 19,13 e $30,38 \mathrm{~kg} / \mathrm{cm}^{2}(M=23,60, D P=3,16)$. Assinale-se que quatro cuidadores tinham duas crianças participantes, respectivamente.

Para o diagnóstico de sobrepeso ou obesidade em crianças, é utilizado o IMC de acordo com a idade e o sexo. Os dados são comparados com as informações das curvas e tabelas de referência do National Center for Health Statistics - NCHS (CDC, 2000). Os resultados obtidos em percentil são considerados diagnóstico de obesidade se o IMC for $\geq$ ao percentil 95 e sobrepeso quando o IMC indicar percentil entre 85 e 95 .

As crianças deste estudo foram selecionadas a partir do percentil: todas estavam com percentil acima de 85 , o que indica sobrepeso. No entanto, para padronização dos dados utilizou-se o dado de IMC peso $(\mathrm{kg})$ /altura x altura(m2), tal como nos adultos.

\section{Materiais}

Foram utilizados balança antropométrica da marca Welmy (Sta Barbara D'Oeste, SP), com capacidade de 140 $\mathrm{kg}$, e Inventários ASEBA: ASR e CBCL. Esses instrumentos foram traduzidos sob a coordenação da Profa. Dra. Edwiges F. M. Silvares, da Universidade de São Paulo, e da Profa. Isabel A. Bordin, da Universidade Federal de São Paulo, com autorização e licença do autor e da editora do instrumento.

Os inventários do ASEBA identificam competências sociais por meio de perguntas que avaliam a rede social do indivíduo - relacionamento com pares e adultos, atividades e passatempos, entre outros - e problemas comportamentais e emocionais. As escalas ASEBA oferecem um sistema de avaliação baseado na descrição de competências sociais e comportamentos adaptativos e não adaptativos informados pelo próprio individuo ou outro informante (pais ou professores). Esses comportamentos são posteriormente agrupados em escalas de síndromes similares às categorias diagnósticas do DSM ponderadas segundo idade e sexo (Bordin et al., 2013).

O CBCL, o ASR e os demais instrumentos do ASEBA dividem os problemas de comportamento em duas categorias: internalizantes e externalizantes. Os problemas de comportamento internalizantes são aqueles encobertos, evidenciados por retraimento, ansiedade e problemas psicossomáticos presentes na depressão e ansiedade infantil patológica. Os problemas externalizantes são caracterizados por serem observáveis, como, por exemplo, impulsividade, agressividade, atitudes desafiadoras e opositoras, frequentes em condutas antissociais e abuso de substâncias (Bordin, Mari, \& Caeiro, 1995; Gauy \& Guimarães, 2006).

O CBCL é uma escala que avalia as competências e comportamentos de crianças e adolescentes com idades entre 6 a 18 anos por meio da informação de um adulto, geralmente os pais. É composto por onze escalas, sendo que três delas avaliam competência social (atividades passatempos, brincadeiras, tarefas diárias; desempenho social - participação em grupos, frequência com que convive com os pares, independência; e desempenho escolar); as demais avaliam problemas de comportamento.
A escala de problemas internalizantes do CBCL engloba três subescalas: ansiedade e depressão, isolamento e depressão e queixas somáticas. As escalas que compõem a avaliação de problemas externalizantes, no CBCL, são: comportamento de quebrar regras e comportamento agressivo. As demais escalas de problemas - sociais, de pensamento e de atenção -, juntamente com as internalizantes e externalizantes, formam o Total de Problemas (Bordin et al., 1995; Gauy \& Guimarães, 2006).

O ASR é o inventário autoaplicável em adultos de 18 a 59 anos. Seu formato é o mesmo do CBCL. No entanto, a média de competências sociais avalia também o relacionamento com companheiro e no trabalho, enquanto a escala de externalizantes inclui comportamento intrusivo (provocar os outros, chamar a atenção para si, falar muito alto).

Nos instrumentos ASEBA, os escores brutos são transformados em T-Scores Esses escores comparam a pontuação do indivíduo com a distribuição dos pontos obtida a partir da amostra normativa.

\section{Procedimento}

A pesquisa foi aprovada pelo Comitê de Ética segundo a Resolução 196/96 da CONEP (Comissão Nacional de Ética em Pesquisas de 1996), de acordo com o parecer no. 554.845. O programa foi conduzido mediante a autorização das famílias, através de assinaturas de Termos de Consentimento Livre e Esclarecido (TCLE), com detalhes dos informes da pesquisa.

Após autorização da escola, foi enviada uma carta informativa sobre o programa multidisciplinar de excesso de peso que iria envolver profissionais de áreas da Medicina, Nutrição, Psicologia e Fisioterapia. Nessa carta, era solicitada a permissão para que a criança fosse pesada e medida. A mensuração de peso e medida foi realizada por estudantes de Nutrição. Depois da mensuração do peso e altura das crianças na escola, novas cartas foram enviadas aos cuidadores dos alunos cujo peso estava em excesso. Essas cartas convidavam os cuidadores para também serem pesados e medidos.

Cuidadores e crianças que estavam com excesso de peso foram convidados a participar do projeto de intervenção multidisciplinar para excesso de peso e consentirem a participação assinando o TCLE. Os participantes não estavam sendo submetidos a tratamentos nutricionais ou psicológicos para excesso de peso. Apenas uma criança fazia consultas regulares com endocrinologista.

Os inventários foram aplicados nos cuidadores, antes de serem submetidos ao programa, após estes assinarem o TCLE. Eles foram informados sobre a importância de responder aos inventários para fins da pesquisa, e foi-lhes assegurado que suas identidades seriam preservadas.

O programa de intervenção multidisciplinar para excesso de peso se desenvolvia uma vez por semana, na escola das crianças, após o período das aulas. O programa, de dez sessões semanais, incluía: orientações nutricionais, realizadas por meio de palestras e dinâmicas de grupo, por concluintes do curso de Nutrição; palestras psicoeducativas, com uso de técnicas terapêuticas comportamentais, conduzidas por psicóloga e alunas de iniciação científica; consultas médicas 
e palestras sobre implicações da obesidade, feitas por médico endocrinologista, com o apoio de alunas de enfermagem; e programa de atividade física, com uso de dinâmicas de grupo, para os escolares, dirigido por alunas concluintes do curso de fisioterapia. A adesão a este estudo foi considerada como presença em pelo menos $70 \%$ dos encontros da intervenção multidisciplinar.

Após a aplicação dos instrumentos (CBCL e ASR), a avaliação dos dados foi realizada por meio do software ADM (Software Assessment Data Manager) desenvolvido por Achenbach e Rescola (2001). Esse software, após o registro das respostas dos inventários, transforma os dados brutos em T-Scores e classifica os indivíduos quanto aos problemas apresentados. Assim, T-Score de 50 a 64 na escala de problemas e de 65 a 69 na escala de normalidade aponta índice limítrofe (situação em que o problema merece atenção, apesar de ainda não ser grave), e de 70 a 100 indica que os problemas são clínicos, ou seja, graves.

\section{Análise dos Dados}

Para análise estatística, foi utilizado o programa SPSS 18 (Statistical Package for Social Sciences). Para avaliar as relações entre problemas de comportamento e competência social de cuidadores e IMC e adesão das crianças, utilizouse o estudo de correlação por meio da técnica de Pearson. A escolha dessa técnica se deveu ao fato de se estar trabalhando com variáveis escalares. Para análise relacional, optou-se por utilizar as pontuações brutas no CBCL e não as ponderadas, a fim de se garantir o uso de estatística paramétrica (Hair Jr., Black, Babin, \& Anderson, 2006). Tomou-se como intervalo de confiança p menor ou igual a 0,05 .

\section{Resultados}

As crianças apresentaram maior número de casos internalizantes clínicos: das 26 crianças, 14 estavam nessa condição e cinco indicaram condição limítrofe. Em relação aos problemas externalizantes, foram observados nove casos clínicos e dois limítrofes.

A Tabela 1 apresenta as correlações entre (a) problemas de comportamento, competência social e IMC das crianças, (b) problemas de comportamento, competência dos cuidadores e IMC das crianças, além de (c) correlação entre problemas de comportamento de crianças e cuidadores e adesão.

Verifica-se, de acordo com os dados apresentados na Tabela 1, relação significativa entre problemas internalizantes das crianças e seu IMC $(r=0,50, p=0,01)$. Quanto mais problemas internalizantes percebidos pelos pais, maior o IMC da criança. Não se observou, porém, relação significativa entre a competência social das crianças relatada pelos cuidadores e o IMC ( $r=-0,31, p=0,15)$.

Tampouco foi observada relação significativa entre adesão ao tratamento e as demais variáveis: competência social das crianças $(r=-0,11, p=0,68)$, competência dos cuidadores $(r=0,31, p=0,13)$, problemas internalizantes das crianças $(r=-0,13, p=0,53)$ e dos cuidadores $(r=-0,23$, $p=0,25)$, problemas externalizantes das crianças $(r=0,14$, $p=0,49)$ e dos cuidadores $(r=-0,21, p=0,29)$, e total de problemas em crianças $(r=0,11, p=0,64)$ e cuidadores $(r$ $=-0,19, p=0,34)$.

Os problemas de comportamento dos cuidadores apareceram relacionados significativamente com os problemas de comportamento das crianças. Mais especificamente, quanto maior o autorrelato de problemas externalizantes dos cuidadores, maior a observação de problemas internalizantes em seus filhos $(r=0,54, p=0,00)$, como também externalizantes $(r=0,54, p=0,01)$ e no total de problemas $(r=0,56$ e $p=0,00)$. De forma semelhante, quanto mais problemas internalizantes os pais apresentam, mais relatos de problemas em seus filhos são da mesma natureza $(r=0,68$ e $p=0,00)$, externalizantes $(r=0,68$ e $p=$ $0,00)$ ou totais $(r=0,69$ e $p=0,00)$.

Os dados apontam, dessa forma, correlação entre os problemas de comportamento dos cuidadores e das crianças. Estes demonstram, também, correlação com alto IMC (ver Figura 1). Nesse sentido, é possível afirmar que os problemas de comportamento das crianças seja uma variável mediacional entre o problema de comportamento dos cuidadores e o IMC das crianças

\section{Discussão}

O presente estudo buscou avaliar relações entre problemas de comportamento e competências de cuidadores, de crianças e IMC destas, para o melhor entendimento da relação entre os problemas de comportamento/competências e excesso de

Tabela 1. Correlação Pearson entre competências sociais e problemas de comportamento de crianças em tratamento para obesidade e IMC das crianças, adesão ao tratamento e competência social e problemas de comportamento de cuidadores.

\begin{tabular}{|c|c|c|c|c|c|c|}
\hline & $\begin{array}{l}\text { Competência } \\
\text { social das } \\
\text { crianças }\end{array}$ & $\begin{array}{c}\text { Problemas } \\
\text { internalizantes } \\
\text { das crianças }\end{array}$ & $\begin{array}{c}\text { Problemas } \\
\text { externalizantes } \\
\text { das crianças }\end{array}$ & $\begin{array}{c}\text { Total de } \\
\text { problemas } \\
\text { das crianças }\end{array}$ & Adesão & $\begin{array}{l}\text { IMC das } \\
\text { crianças }\end{array}$ \\
\hline Poblemas externalizantes dos cuidadores & $-0,03$ & $0,54 * *$ & $0,54 * *$ & $0,56 * *$ & 0,14 & 0,01 \\
\hline Problemas internalizantes dos cuidadores & $-0,03$ & $0,68 * *$ & $0,56 * *$ & $0,69 * *$ & $-0,23$ & 0,11 \\
\hline Total de problemas dos cuidadores & 0,14 & $0,57 * *$ & $0,57 * *$ & $0,60 * *$ & $-0,19$ & 0,09 \\
\hline Competência social dos cuidadores & 0,01 & $-0,25$ & $-0,16$ & $-0,24$ & $-0,11$ & 0,87 \\
\hline Adesão & 0,11 & $-0,13$ & 0,14 & 0,11 & 1 & $\mathrm{XX}$ \\
\hline IMC & $-0,31$ & $0,50 *$ & $-0,07$ & 0,14 & XX & 1 \\
\hline
\end{tabular}

Nota:* Correlação significativa no nível de $0,05 . * *$ Correlação significativa no nível de 0,01 . 


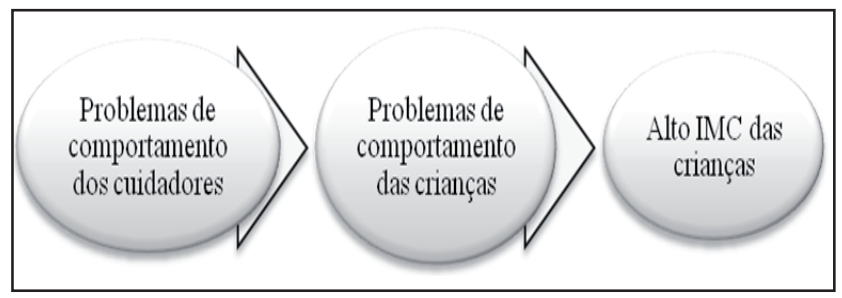

Figura 1. Relação entre problemas de comportamento dos cuidadores e problemas de comportamento das crianças, e destes últimos com o IMC

peso. Todos participaram de um programa de intervenção multidisciplinar.

Os resultados demonstraram relação significativa entre problemas internalizantes - percebidos pelos cuidadores - e IMC $(r=0,50, p=0,01)$ nas crianças estudadas. Pode-se dizer que esse resultado assemelha-se aos de vários estudos que utilizaram o mesmo instrumento e apontaram problemas de comportamento na população com excesso de peso (Braet et al., 1997; Israel \& Shapiro, 1985; Luiz et al., 2010; Sousa $\&$ Moraes, 2011).

Luiz et al. (2010), assim como Sousa e Moraes (2011), observaram que os comportamentos internalizantes prevalecem sobre os externalizantes em crianças com excesso de peso. Os resultados da presente pesquisa corroboram essa constatação, pois os comportamentos internalizantes mostraram-se mais constantes entre os participantes do estudo.

Buscou-se também verificar a relação entre esses problemas/competências e a adesão (presença em 70\% dos encontros) das crianças. Não foram encontradas relações significativas entre a adesão destas e problemas de comportamento e competência de cuidadores e crianças.

Relações independentes entre problemas de comportamento dos cuidadores e IMC das crianças foram percebidas. Contudo, verificaram-se relações entre problemas de comportamento dos cuidadores e problemas de comportamento das crianças. Desse modo, os resultados do presente estudo sugerem que os problemas de comportamento das crianças sejam uma variável mediacional, isto é, aquela que, por meio da variável independente (no caso, problemas de comportamento dos cuidadores), influencia a variável dependente (IMC das crianças) (Baron \& Kenny, 1986). Novos estudos devem ser realizados para melhor analisar o papel dessa variável mediacional.

Segundo Sanger, MacLean e Slyke (1992), os problemas de comportamento dos cuidadores demonstram relacionar-se com os problemas de comportamento das crianças. O estudo de Myers et al. (1998) com uma população de crianças com idades semelhantes às do presente estudo, mas com uma amostra maior (116), além de encontrar associação entre os problemas de comportamento de pais e filhos, mostrou que melhoras no estado psicológico das mães estavam relacionadas a melhoras no ajustamento psicossocial dos filhos em todas as escalas de problemas, fator que também repercutia em efeitos na perda de peso das crianças.

Os resultados apontam para a necessidade de abordar os problemas de comportamento, tanto de cuidadores como de crianças, nas intervenções que visam tratar o problema do excesso de peso. Contudo, dadas as características da amostra - pequena, selecionada por conveniência e não probabilística -, os dados não podem ser generalizados. $\mathrm{O}$ fato de ter sido selecionado apenas um informante (um cuidador) também limitou os apontamentos deste estudo. Entretanto, apesar dessas limitações, o presente estudo põe em realce a existência de relação entre problemas de comportamento e IMC aumentado, sugerindo que o trabalho com crianças obesas (ou com IMC alto) deve também levar em consideração aspectos psicológicos e comportamentais que podem estar associados. Estudos com um desenho metodológico diferente do apresentado neste artigo, com um maior número de participantes, com intervenções na construção de repertórios de comportamentos habilidosos, com grupo controle e utilização de inventários autoavaliativos poderiam ajudar a esclarecer melhor como essa relação se estabelece.

\section{Referências}

Achenbach, T. M., \& Rescorla, L.A. (2001). Manual for the ASEBA school-age forms \& profiles. Burlington, VT: University of Vermont, Department of Psychiatry.

Achenbach, T. M., \& Rescorla, L.A. (2003). Manual for the ASEBA adult forms \& profiles. Burlington, VT: University of Vermont, Department of Psychiatry.

Anderson, S. E., He, X., Schoppe-Sullivan, S., \& Must, A. (2010). Externalizing behavior in early childhood and body mass index from age 2 to 12 years: Longitudinal analysis of a prospective cohort study. BioMed Central Pediatrics, 49, 1-10. Doi: 10.1186/1471-2431-10-49

Baile, J. J., \& González, M. J. (2011). Comorbilidad psicopatológica en obesidad. Anales del Sistema Sanitario de Navarra, 34(2), 253-261.

Baron, R. M., \& Kenny, D. A. (1986). The moderator-mediator variable distinction in social psychological research: Conceptual, strategic, and statistical considerations. Journal of Personality \& Social Psychology, 51, 1173-1182. Doi: 10.1037/0022-3514.51.6.1173

Bordin, I. A. S., Mari, J. J., \& Caeiro, M. F. (1995). Validação da Versão Brasileira do Child Behavior Checklist [CBCL]; Inventário de comportamento da infância e da adolescência: Dados preliminares. Revista da Associação Brasileira de Psiquiatria, 17(2), 55-66.

Bordin, I. A. S., Rocha, M. M., Paula, C. S., Teixeira, M. C. T. V., Rescorla, A. L., \& Silvares, E. F. M. (2013). Child Behavior Checklist (CBCL), Youth Self-Report (YSR) and Teacher's Report Form (TRF): An overiew of the development of the original and Brazilian versions. Caderno de Saúde Pública, 29(1), 13-28.

Braet, C., Mervielde, I., \& Vandereycken, W. (1997). Psychological aspects of childhood obesity: A controlled study in a clinical and nonclinical Sample. Journal of Pediatric Psychology, 22, 59-71.Doi: 10.1093/jpepsy/22.1.59

Epstein, L.H., Wisniewski, L., \& Weng, R. (1994). Child and parent psychological problems influence child weight control. Obesity Research, 2, 509-51. Doi: 10.1002/j.1550-8528.1994. tb00099.x 
Epstein, L. H., Valoski, A., Wing, R. R., \& McCurley, J. (1990). Tenyear follow-up of behavioral, family-based treatment for obese children. The Journal of the American Medical Association, 264(19), 2519-2528. Doi: 10.1001/jama.264.19.2519

Friedman, M. A., \& Brownell, K. D. (1995). Psychological correlates of obesity: Moving to the next research generation. Psychological Bulletin, 11(7), 3-20. Doi: 10.1037/00332909.117.1.3

Gauy, F. V., \& Guimarães, S. S. (2006). Triagem em saúde mental infantil. Psicologia: Teoria e Pesquisa, 22, 05-16.

Hair Jr., J. F., Black, W. C., Babin, B.J., \& Anderson, R.E. (2006). Multivariate data analysis.( 7a ed.) Upper Saddle River: Pearson Prentice Hall.

Haavisto, A., Jalanko, H., Sintonen, H., Holmberg, C. \& Qvist, E. (2006). Quality of life in adult survivors of pediatric kidney transplantation. Transplantation, 92, 1322-1326. Doi: 10.1097/ TP.0b013e318237062b

Israel, A. C., \& Shapiro, L. S. (1985). Behavior problems of obese children enrolling in a weight reduction program. Journal of Pediatric Psychology, 10(4), 449- 460. Doi: 10.1093/ jpepsy/10.4.449

Luiz, A. M. A. G., Gorayeb, R., \& Liberatone, R. D. R. (2010). Avaliação de depressão, problemas de comportamento e competência social em crianças obesas. Estudos de Psicologia, 27(1), 41-48.

Myers, M. D., Hollie, M. A., Raynor, R. D., \& Epstein, L H. (1998). Predictors of child psychological changes during family-based treatment for obesity. Archives of Pediatrics and Adolescent Medicine Journal, 152, 855-861. Doi: 10.1001/ archpedi.152.9.855
Napolitano, M. A., \& Hayes, S. (2011) Behavioral and psychological factors associated with 12-month weight change in a physical activity trial. Journal of Obesity, 1-10. Doi: 10.1155/2011/515803

Retz, M., W., Thome, J., Schneider, M., Stieglitz, R. D., \& Falkai, P. (2008). Psychopathological rating scales for diagnostic use in adults with attention-deficit/hyperactivity disorder (ADHD). European Archives of Psychiatry and Clinical Neuroscience, 256, 3-11

Sanger, M. S., MacLean, W. E., \& Slyke, V. A. D. (1992). Relation between maternal characteristics and child behavior ratings: Implications for interpreting behavior checklists. Clinic Pediatrics, 31, 461-465. Doi: 10.1177/000992289203100803

Sousa, M. R., \& Moraes, C. (2011). Sintomas de internalização e externalização em crianças e adolescentes com excesso de peso. Journal Brasileiro de Psiquiatria, 60(1), 40-45.

Stunkard, A. J., Faith, M. S., \& Allison, K. C.( 2003). Depression and obesity. Biological Psychiatry, 54, 330-337. Doi: 10.1016/ S0006-3223(03)00608-5

Tiffin, P. A., Arnott, B., Moore, H. J., \& Summerbell, C. D. (2011). Modelling the relationship between obesity and mental health in children and adolescents: Findings from the Health Survey for England 2007. Child and Adolescent Psychiatry and Mental Health, 31(5), 1-11. Doi: 10.1186/1753-2000-5-31

Vieira, P. N., Mata, J., Silva, M. N., Coutinho, S. R., Santos, T. C., Minderico, C. S., Sardinha, L. B., \& Teixeira, P. J. (2011). Predictors of psychological well-being during behavioral obesity treatment in women. Journal of Obesity, Article ID 936153.Doi: 10.1155/2011/936153

Wadden, T. A., Foster, G. D., Brownell, K. D., \& Finley, E. (1984). Self-concept in obese and normal weight children. Journal of Consulting and Clinical Psychology, 52, 1104-1105. 